\title{
2.5D SOUND FIELD REPRODUCTION IN HIGHER ORDER AMBISONICS
}

\author{
Wen Zhang and Thushara D. Abhayapala
Research School of Engineering, CECS, The Australian National University, Canberra, Australia Email:\{wen.zhang, thushara.abhayapala\}@anu.edu.au

\begin{abstract}
In this paper, we present a theoretical basis and implementation strategies for 2.5-dimensional (2.5D) sound field reproduction in higher order Ambisonics. The issue associated with $2.5 \mathrm{D}$ system is the intrinsic dimensionality mismatch between a desired 2-dimensional (2D) sound field and the loudspeaker employed for reproduction, whose spatial properties are more accurately modelled by that of a 3-dimensional (3D) point source. We formulate this dimensionality mismatch problem in higher order Ambisonics and show that referencing the desired 2D sound field with the corresponding reproduced sound field at the reproduction centre would give the most accurate results. We also derive analytical solutions using $3 \mathrm{D}$ point sources as secondary sources for both $2 \mathrm{D}$ plane wave and cylindrical wave reproduction. The reproduction accuracy of the proposed method is verified through numerical simulations.
\end{abstract}

Index Terms - 2.5D reproduction, higher order Ambisonics, circular loudspeaker array

\section{INTRODUCTION}

Sound field reproduction in the horizontal plane at a height approximately level with the listener's ears is of great relevance in practical situations. This establishes essentially the $2 \mathrm{D}$ reproduction problem, in which the reproduced sound fields are modelled as height invariant and a natural choice of secondary sources for reproduction would be line sources which exhibit 2D transfer functions [1-3]. However, loudspeakers employed in practice have characteristics to be more accurately modelled by that of a $3 \mathrm{D}$ point source. Because of the intrinsic dimensionality mismatch, using $3 \mathrm{D}$ point sources as secondary sources in 2D reproduction results in artifacts and is termed as $2.5 \mathrm{D}$ reproduction $[4,5]$.

Implementation of $2.5 \mathrm{D}$ wave field synthesis (WFS) using the Kirchhoff-Helmholtz integral [6] is based on two separate stationary phase approximations and hence the reproduction is only accurate for large distances of the virtual source or the listener location to the secondary source positions $[2,4,5]$. Spectral division method (SDM) proposed by Ahrens and Spors [7,8] formulates the 2.5D reproduction equation in wavenumber domain with employment of linear distributions of secondary sources. A characteristic in $2.5 \mathrm{D}$ WFS and SDM reproduction is that the reproduced plane waves exhibit an amplitude decay, approximately $3 \mathrm{~dB}$ per doubling distance from the secondary source distribution $[8,9]$.

Higher order Ambisonics (HOA) formulation is based on circular harmonic [3,10] or spherical harmonic [11-13] decomposition of a sound field and normally referred as "mode-matching" approach. Reproduction of 2D plane waves within a small reproduction region

This work was supported under the Australian Research Councils Discovery Projects funding scheme (project no. DP110103369).
( $0.1 \mathrm{~m}$ radius) using a circular array of $3 \mathrm{D}$ point sources has been implemented in [14]. A spherical harmonic solution to the 2D reproduction problem is proposed based on the sectorial spherical harmonic representation of the plane wave in the horizontal plane [1517]. So far, the $2.5 \mathrm{D}$ performance has only been investigated for synthesis of plane waves; to our best knowledge, no analytical approach has been formulated in 2.5D HOA. Implementation of $2.5 \mathrm{D}$ HOA, however, has great practical significance, especially in combination with recent advances in spatial sound, such as multi-zone sound reproduction [18-20] and employment of higher-order loudspeakers [21,22] to control sound radiation over wide areas.

This paper develops analytical solutions of 2.5D sound field reproduction in higher order Ambisonics. The desired and reproduced sound fields are represented using cylindrical and spherical harmonic decomposition, respectively. We perform a detailed error analysis to demonstrate that referencing the desired sound field with the reproduced sound field at the reproduction centre not only solves the dimensionality mismatch problem but also produces the most accurate results. The proposed 2.5D formula are evaluated through comparison with the $2 \mathrm{D}$ system using line sources and conventional HOA decoding.

\section{THEORY}

A 2D sound field at an observation point $\boldsymbol{x}=\{r, \phi\}$ in a source free region of space can be represented in the following form [1]

$$
S_{d}(\boldsymbol{x}, k)=\sum_{m=-\infty}^{\infty} \alpha_{m}(k) J_{m}(k r) e^{i m \phi},
$$

where $k=2 \pi f / c$ is the wavenumber (with $f$ the frequency and $c$ the speed of wave propagation), $J_{m}(\cdot)$ is the $m$ th order Bessel function of the first kind, and $\alpha_{m}(k)$ are a set of expansion coefficients. For a 2D wave source of unit strength located at $\boldsymbol{y}_{v}=\left\{r_{v}, \phi_{v}\right\}$ with $r_{v}>r$, analytical results of the expansion coefficients can be derived as [23, p. 67]

$$
\alpha_{m}(k)=\left\{\begin{array}{lr}
i^{m} e^{-i m \phi_{v}}, & \text { plane wave } \\
\frac{i}{4} H_{m}^{(2)}\left(k r_{v}\right) e^{-i m \phi_{v}}, & \text { cylindrical wave }
\end{array}\right.
$$

where $H_{m}^{(2)}(\cdot)$ is the $m$ th order Hankel function of the second kind. A rule of thumb is given in [24] for truncating (1) to an order $M=$ $\left\lceil e k r_{0} / 2\right\rceil$ that is sufficient to represent the field within a region of interest, i.e., a spatial region $\mathbb{C}$ within a circle of radius $r_{0}$ (or $\forall r \leq$ $\left.r_{0}\right)$.

Assume that we want to reproduce the sound field represented by (1) within the region of interest (the so called reproduction region) using a circular array of $L$ loudspeakers placed in the horizontal plane. For exact reproduction of a 2D wave up to the $M$ th order, 
the number of loudspeakers required is $L \geq 2 M+1$ [14]. Each loudspeaker is a 3D point source that produces a sound field

$$
T_{\ell}(\boldsymbol{x}, k)=\frac{e^{-i k\left|\boldsymbol{y}_{\ell}-\boldsymbol{x}\right|}}{4 \pi\left|\boldsymbol{y}_{\ell}-\boldsymbol{x}\right|}, \ell=1, \ldots, L
$$

at the observation point $\boldsymbol{x}$; the loudspeaker position in the horizontal plane is specified by $\boldsymbol{y}_{\ell}=\left\{R, \varphi_{\ell}\right\}$ with the same origin $\mathcal{O}$ as the reproduction region and radius $R>r_{0}$.

The sound field due to the loudspeaker array can be written in the following form

$$
S(\boldsymbol{x}, k)=\sum_{\ell=1}^{L} w_{\ell}(k) T_{\ell}(\boldsymbol{x} ; k),
$$

where $w_{\ell}(k)$ is a complex-valued frequency-dependent weight function to the $\ell$ th loudspeaker. Substituting the expansion of (3) $[23$, p. 30$]$ into (4) then leads to the expression

$$
\begin{aligned}
S(\boldsymbol{x}, k)=\sum_{n=0}^{\infty} \sum_{m=-n}^{n} & -i k j_{n}(k r) h_{n}^{(2)}(k R) \\
& \times \sum_{\ell=1}^{L} w_{\ell}(k) Y_{n m}^{*}\left(\hat{\boldsymbol{y}}_{\ell}\right) Y_{n m}(\hat{\boldsymbol{x}}),
\end{aligned}
$$

where $j_{n}(\cdot)$ and $h_{n}^{(2)}(\cdot)$ are the first kind spherical Bessel function and second kind spherical Hankel function of the $n$th degree, respectively. In the spherical coordinate system, the unit vectors in the horizontal plane are denoted by $\hat{\boldsymbol{x}}=\{\pi / 2, \phi\}$ and $\hat{\boldsymbol{y}}_{\ell}=\left\{\pi / 2, \varphi_{\ell}\right\}$; both elevation angles are equal to $\pi / 2$. (.)* denotes the complex conjugate operation.

The spherical harmonics are defined as [25, p. 190-193]

$$
Y_{n m}(\theta, \phi)=A_{n m} P_{n m}(\cos \theta) E_{m}(\phi),
$$

where

$$
A_{n m}=\sqrt{\frac{2 n+1}{2} \frac{(n-|m|) !}{(n+|m|) !}},
$$

$P_{n m}(\cdot)$ is the associated Legendre function, and the normalised exponential function $E_{m}(\phi)=e^{i m \phi} / \sqrt{2 \pi}$.

By substituting (5) with the spherical harmonics at the elevation of $\pi / 2$ and changing the summation order, we have

$$
\begin{aligned}
S(\boldsymbol{x}, k)= & \sum_{m=-\infty}^{\infty} \sum_{n=|m|}^{\infty}-i k j_{n}(k r) h_{n}^{(2)}(k R) \\
& \times A_{n m}^{2} P_{n m}^{2}(0) E_{m}(\phi) \sum_{\ell=1}^{L} w_{\ell}(k) E_{-m}\left(\varphi_{\ell}\right) .
\end{aligned}
$$

Equating (1) with (8) up to the $M$ th mode, multiplying both sides by $e^{-i q \phi}$ and integrating over the unit circle with respect to $\phi$ gives

$$
\begin{array}{r}
\alpha_{m}(k) J_{m}(k r)=\beta_{m}(k) Q_{m}(k r, k R), \\
m=-M, \ldots, M
\end{array}
$$

where the derivation is based on the orthogonality property of complex exponentials, i.e.,

$$
\int_{0}^{2 \pi} E_{m}(\phi) E_{-q}(\phi) d \phi=\delta_{m q},
$$

and the following definitions

$$
\begin{aligned}
Q_{m}(k r, k R) & =\sum_{n=|m|}^{M}-i k j_{n}(k r) h_{n}^{(2)}(k R) A_{n m}^{2} P_{n m}^{2}(0) \\
\beta_{m}(k) & =\sum_{\ell=1}^{L} w_{\ell}(k) E_{-m}\left(\varphi_{\ell}\right) .
\end{aligned}
$$

The general form to reproduce a desired sound field is to design the loudspeaker weights as least-square solutions to (12), where $\beta_{m}(k)$ known as Fourier series coefficients of the loudspeaker driving functions ${ }^{1}$ are determined by

$$
\beta_{m}(k)=\frac{\alpha_{m}(k) J_{m}(k r)}{Q_{m}(k r, k R)}, m=-M, \ldots, M .
$$

A special case is when the loudspeakers are equally spaced in the circular array, we have

$$
w_{\ell}(k)=\frac{2 \pi}{L} \sum_{m=-M}^{M} \beta_{m}(k) E_{m}\left(\varphi_{\ell}\right),
$$

where $\varphi_{\ell}=2 \pi(\ell-1) / L, \ell=1, \ldots, L$.

\section{SOLUTION}

The reproduced sound field has to be referenced with the desired sound field at a particular radius $r=r_{\text {ref }}$ to determine the coefficients $\beta_{m}(k)$. There is the situations that the solution is undefined, i.e., the summation of spherical Bessel function over degree $n$ at the denominator of (13) goes to zero when $k r \neq 0$. Given complicated mathematical expressions appeared at the numerator and denominator of (13), letting $r_{\text {ref }}$ tend to zero as in conventional Ambisonics has been adopted in the sectorial spherical harmonic based approach [15]. In this section, we firstly give detailed error analysis to demonstrate this choice would result in the minimum 2.5D reproduction error and then derive an analytical expression for the coefficients of the loudspeaker driving functions.

\subsection{Error Analysis}

The relative mode-matching error over the entire reproduction region $\mathbb{C}$ can be written as

$$
\begin{aligned}
& e\left(r_{\text {ref }}, k\right) \\
& =\int_{0}^{r_{0}} \sum_{m=-M}^{M} \frac{\left|\alpha_{m}(k) J_{m}(k r)-\beta_{m}(k) Q_{m}(k r, k R)\right|^{2}}{\left|\alpha_{m}(k) J_{m}(k r)\right|^{2}} r d r \\
& =\int_{0}^{r_{0}} \sum_{m=-M}^{M}\left|1-\frac{J_{m}\left(k r_{\text {ref }}\right) Q_{m}(k r, k R)}{J_{m}(k r) Q_{m}\left(k r_{\text {ref }}, k R\right)}\right|^{2} r d r
\end{aligned}
$$

Plot in Fig. 1(a) demonstrates the general trend that the matching error increases with the reference radius $r_{\text {ref }}$ and becomes substantial above a certain value even within the reproduction region. We should keep $r_{\text {ref }}$ within a small range around the reproduction centre and this range shrinks as the operating frequency increases. Choosing the reference point at the reproduction centre can always guarantee the minimum reproduction error.

\footnotetext{
${ }^{1}$ This is to represent the loudspeaker driving signals as spatial samples of a continuous loudspeaker aperture function, which is a periodic function of angle $\varphi$ and can be expanded by the Fourier series [3].
} 


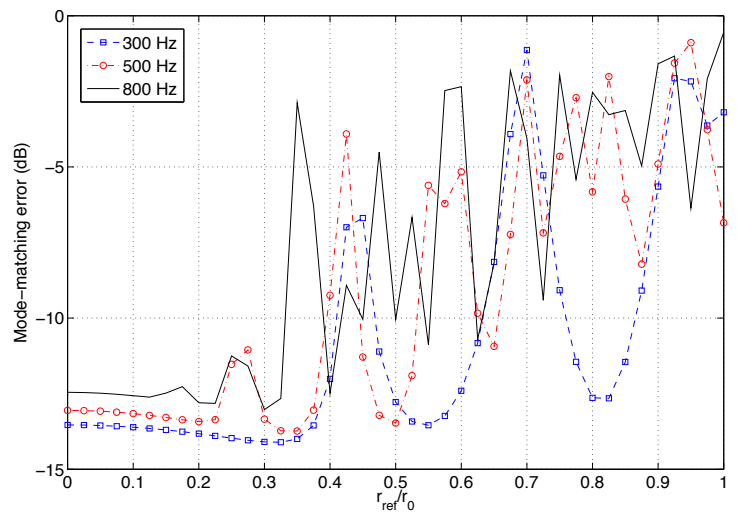

(a)

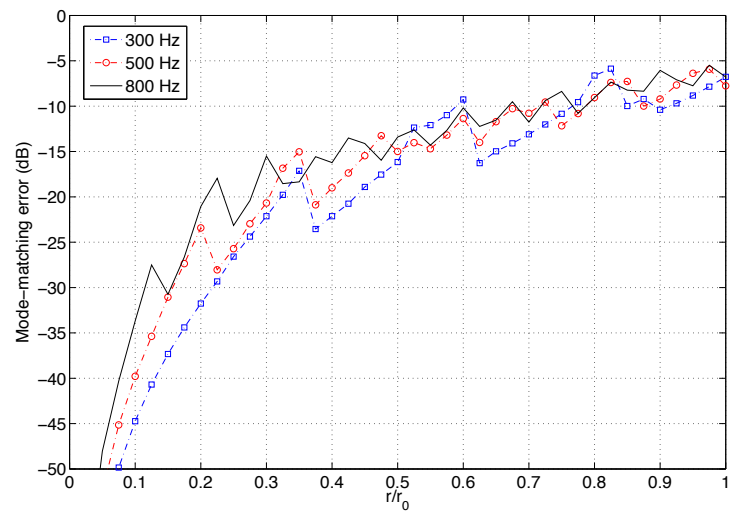

(b)

Fig. 1. Mode-matching error at (a) different reference radius $r_{\text {ref }}$ or (b) observation positions $r$ when $r_{\text {ref }}=0$.

\subsection{Analytical Solutions}

The derivation is based on application of L'Hôpital's rule [26, p. 13] and the following identities [25, p. 118, 196],

$$
\begin{aligned}
& j_{n}(k r) \approx \frac{(k r)^{n}}{(2 n+1) ! !} \\
& J_{m}(k r) \approx \frac{\left(\operatorname{sgn}(m)^{|m|}\right)(k r)^{|m|}}{2^{|m|}|m| !}
\end{aligned}
$$

for the small arguments, i.e., $k r \rightarrow 0$. Note that the summation over $n$ in (11) reduces to a single term with $n=|m|$. Then, we yield an analytical expression for the coefficients,

$$
\beta_{m}(k)=\frac{C_{m} \alpha_{m}(k)}{-i k h_{|m|}^{(2)}(k R) A_{|m| m}^{2} P_{|m| m}^{2}(0)},
$$

where

$$
C_{m}=\frac{\left(\operatorname{sgn}(m)^{|m|}\right)(2|m|+1) ! !}{2^{|m|}|m| !}
$$

and $\operatorname{sgn}(\cdot)$ denotes the sign function.
Substituting (17) into (8) then leads to the following representation of the reproduced sound field

$$
S_{r}(\boldsymbol{x}, k)=\sum_{m=-M}^{M} \alpha_{m}(k) e^{i m \phi} \frac{C_{m} Q_{m}(k r, k R)}{-i k h_{|m|}^{(2)}(k R) A_{|m| m}^{2} P_{|m| m}^{2}(0)} .
$$

As the reference point is set at the reproduction centre, exact sound field reproduction is achieved only at this point. The mode-matching error monotonically increases as the observation position changes from the centre towards the boundary of the reproduction region as shown in Fig. 1(b). The error also slightly increases with the operating frequency.

\section{SIMULATION RESULTS}

To check the performance of the proposed scheme, examples of a circular array of loudspeakers placed in the horizontal plane for 2D plane wave and cylindrical wave reproduction are examined. We define the reproduction error as the average squared difference between the desired field and corresponding reproduced field,

$$
\varepsilon(k r)=10 \log _{10} \frac{\int_{\mathbb{C}}\left|S_{d}(\boldsymbol{x}, k)-S_{r}(\boldsymbol{x}, k)\right|^{2} d \boldsymbol{x}}{\int_{\mathbb{C}}\left|S_{d}(\boldsymbol{x}, k)\right|^{2} d \boldsymbol{x}} .
$$

In the first example, we simulate a monochromatic 2D plane wave and a cylindrical wave of frequency $500 \mathrm{~Hz}$, incident from $\phi_{v}=\pi / 2$ (and $r_{v}=4 \mathrm{~m}$ for the cylindrical wave source), within a $2 \mathrm{D}$ reproduction plane of radius $r_{0}=1 \mathrm{~m}$. This gives $k r_{0}=9.16$, and the rule of thumb suggests using $M=\left\lceil e k r_{0} / 2\right\rceil=13$, thus requiring at least $L=27$ loudspeakers. The loudspeakers are equiangularly placed on a circle of radius $R=3 \mathrm{~m}$, with locations shown as " $x$ " marks in Fig. 2. The reproduced sound fields using 2D line sources [3], 3D point sources with the proposed reference at the reproduction centre, and the conventional HOA decoding are displayed. The 2D line sources are theoretical solutions to this problem thus provide the best results; however it is impractical to build infinite-long line sources for implementation. Both the proposed scheme and the conventional HOA with the reference at the reproduction centre produce undistorted wave fronts. The conventional HOA decoding however based on modelling loudspeakers as plane wave sources produces the worst reproduction performance as the loudspeaker distance information and its generated spherical wavefronts are not matched. The proposed scheme, on the other hand, produce comparable results as the theoretical 2D system in the $x-y$ plane. As shown in Fig. 3, the reproduction error in the 2.5D system is higher than that in the $2 \mathrm{D}$ case.

We next investigate the performance of the proposed scheme for reproducing sound fields at various frequencies and radii. Figure 3 compares the 2D, 2.5D and conventional HOA reproduction error versus $k r$ for a plane wave from $\phi_{v}=\pi / 2$ and a cylindrical wave from the same directions and located at $r_{v}=4 \mathrm{~m}$. Overall, the conventional HOA system has the largest error, which proves that it is more appropriate to model the loudspeaker as a point source rather than a plane wave source; the reproduction performance of the proposed 2.5D system sits between the conventional HOA system and the theoretical 2D system. We note that the system designed at a particular frequency and radius $(k r=9.16$ in the example) can provide accurate sound field reproduction for numbers less than the designed value. At the large $k r$ beyond the designed value, the reproduction error becomes significant and monotonically increases with $k r$. This again validates the rule of thumb proposed in [24] for choosing the required truncation order of (1). 

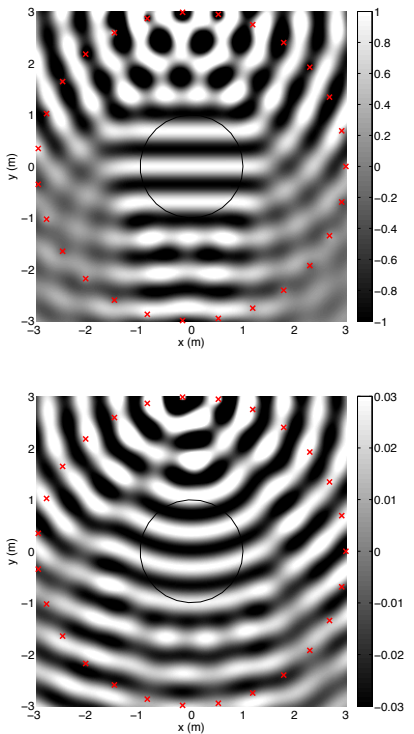

(a) $2 \mathrm{D}$
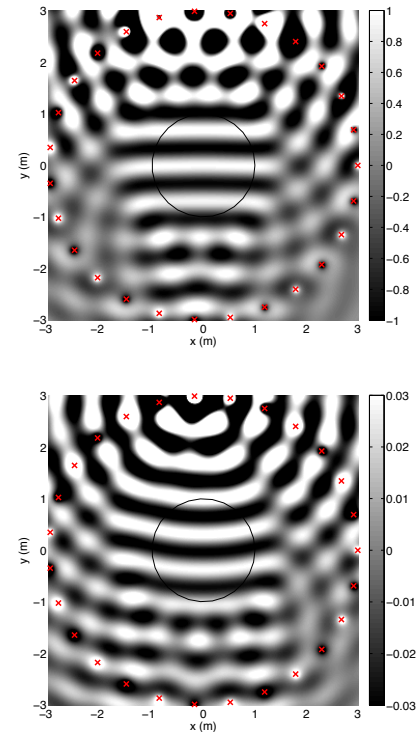

(b) $2.5 \mathrm{D}$ with $r_{\text {ref }}=0$
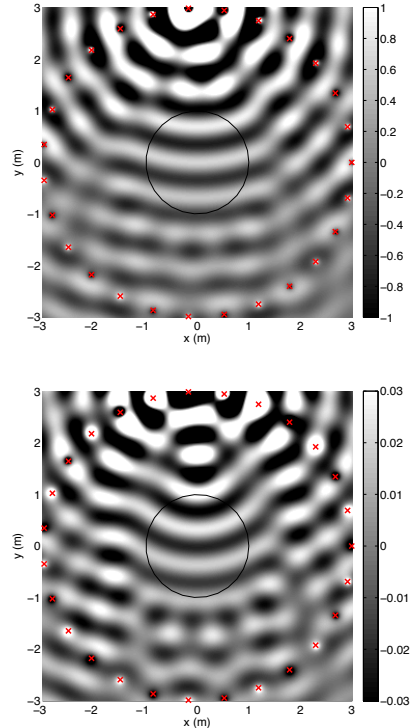

(c) HOA decoding

Fig. 2. Reproduction of a 2D plane wave from $\phi_{v}=\pi / 2$ (upper row) and a cylindrical wave from $r_{v}=4 \mathrm{~m}, \phi_{v}=\pi / 2$ (lower row) for reproduction region of radius $1 \mathrm{~m}$ and frequency of $500 \mathrm{~Hz}$. The loudspeakers are equally placed on a circle of radius $R=3 \mathrm{~m}$, with locations shown as " $\mathrm{x}$ " marks. The encircled region is the reproduction region.

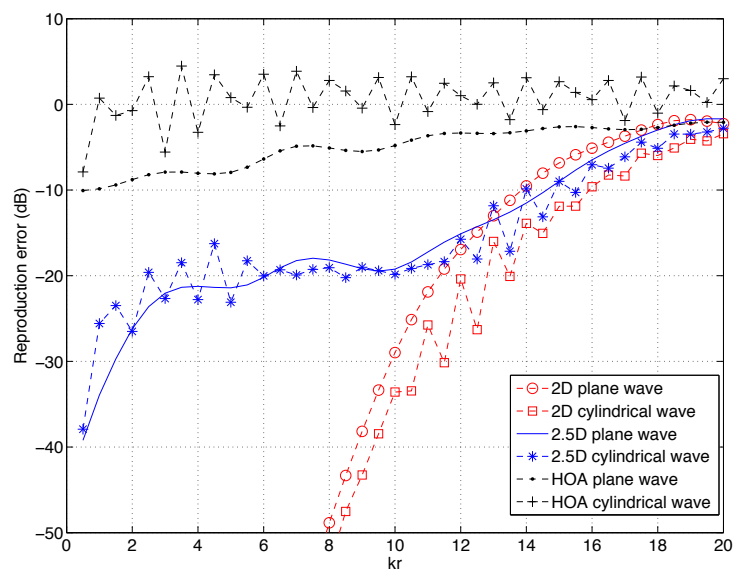

Fig. 3. Comparison of 2D, 2.5D and conventional HOA reproduction error as a function of $k r$.

\section{CONCLUSION}

This paper has considered the modelling of a loudspeaker as a 3D point source and the design strategies to employ a circular loudspeaker array for 2.5D sound field reproduction based on the higher order Ambisonic approach. Here, we have used a cylindrical harmonic expansion of the desired sound field and a spherical harmonic expansion of the sound field generated by the loudspeaker array. To solve the intrinsic dimensionality mismatch problem, we used the design of referencing the desired sound field with the synthesised sound field at the reproduction centre and derived analytical solutions of 2.5D sound field reproduction in higher order Ambisonics. Simulation results demonstrated that both $2 \mathrm{D}$ plane waves and cylindrical waves can be accurately reproduced using the proposed method.

\section{REFERENCES}

[1] M. A. Poletti, "A unified theory of horizontal holographic sound system," Journal of the Audio Engineering Society, vol. 48, no. 12, pp. 1155-1182, 2000.

[2] S. Spors, R. Rabenstein, and J. Ahrens, "The theory of wave field synthesis revisited," in Proc. 124th Convention of the Audio Engineering Society, Amsterdam, Netherlands, May 2008.

[3] Y. Wu and T. D. Abhayapala, "Theory and design of soundfield reproducion using continuous loudspeakers concept," IEEE Transactions on Audio, Speech, and Language Processing, vol. 17, no. 1, pp. 107-116, 2009.

[4] E. W. Start, Direct Sound Enhancement by Wave Field Synthesis, Ph.D. thesis, Delft University of Technology, 1997.

[5] S. Spors and J. Ahrens, "Analysis and improvement of preequalization in 2.5-dimensional wave field synthesis," in Proc. 128th Convention of the Audio Engineering Society, London, UK, May 2010.

[6] A. J. Berkhout, D. de Vries, and P. Vogel, "Acoustic control by wave field synthesis," Journal of the Acoustical Society of America, vol. 93, no. 5, pp. 2764-2778, 1993.

[7] J. Ahrens and S. Spors, "Sound field reproduction using planar and linear arrays of loudspeakers," IEEE Transactions on Audio, Speech and Language Processing, vol. 18, no. 8, pp. 2038-2050, 2010. 
[8] J. Ahrens and S. Spors, "Applying the ambisonics approach to planar and linear distributions of secondary sources and combinations thereof," Acta Acustica united with Acustica, vol. 98, pp. 28-36, 2012.

[9] J. Ahrens, The Single-layer Potential Approach Applied to Sound Field Synthesis Including Cases of Non-enclosing Distributions of Secondary Sources, Ph.D. thesis, Technische Universität Berlin, 2010.

[10] M. A. Poletti, "Robust two-dimensional surround sound reproduction for nonuniform loudspeaker layouts," Journal of the Audio Engineering Society, vol. 55, no. 7/8, pp. 598-610, 2007.

[11] M. A. Poletti, "Three-dimensional surround sound systems based on spherical harmonics," Journal of the Audio Engineering Society, vol. 53, no. 11, pp. 1004-1025, 2005.

[12] F. M. Fazi, P. A. Nelson, J. E. N. Christensen, and J. Seo, "Surround system based on three-dimensional sound field reconstruction," in Proc. 125th Convention of the Audio Engineering Society, San Francisco, CA, USA, Oct. 2008.

[13] H. Sun, S. Yan and U. P. Svensson, "Optimal higher order ambisonics encoding with predefined constraints," IEEE Transactions on Audio, Speech, and Language Processing, vol. 20, no. 3, pp. 742-754, 2011.

[14] D. B. Ward and T. D. Abhayapala, "Reproduction of a planewave sound field using an array of loudspeakers," IEEE Transactions on Speech and Audio Processing, vol. 9, no. 6, pp. 697707, 2001.

[15] J. Ahrens and S. Spors, "An analytical approach to sound field reproduction using circular and spherical loudspeaker distributions," Acta Acustica united with Acustica, vol. 94, pp. 988999, 2008.

[16] J. Ahrens and S. Spors, "Analytical driving functions for higher order ambisonics," in Proc. of the IEEE International Conference on Acoustics, Speech, and Signal Processing, ICASSP 2008, Las Vegas, Nevada, April 2008, pp. 373-376.

[17] M. A. Poletti, T. Betlehem, and T. D. Abhayapala, "Analysis of 2D sound reproduction with fixed-directivity loudspeakers," in Proc. IEEE International Conference on Acoustics, Speech, and Signal Processing, ICASSP 2012, Kyoto, Japan, March 2012, pp. 377-380.

[18] T. Betlehem and C. Withers, "Sound field reproduction with energy constraint on loudspeaker weights," IEEE Trans. Audio, Speech, and Language Process., vol. 20, no. 8, pp. 23882392, 2012.

[19] Y. Wu and T. D. Abhayapala, "Spatial multizone soundfield reproduction: Theory and design," IEEE Transactions on Audio, Speech, and Language Processing, vol. 19, no. 6, pp. 1711$1720,2011$.

[20] J-H. Chang and F. Jacobsen, "Sound field control with a circular double-layer array of loudspeakers," Journal of the Acoustical Society of America, vol. 131, no. 6, pp. 4518-4525, 2012.

[21] M. A. Poletti and T. D. Abhayapala, "Spatial sound reproduction systems using higher order loudspeakers," in Proc. IEEE International Conference on Acoustics, Speech and Signal Processing, ICASSP 2011, Prague, Czech Republic, May 2011, pp. 57-60.

[22] M. A. Poletti, T. D. Abhayapala, and P. Samarasinghe, "Interior and exterior sound field control using two dimensional higher-order variable-directivity sources," Journal of the Acoustical Society of America, vol. 131, no. 5, pp. 3814-3823, 2012.

[23] D. Colton and R. Kress, Inverse Acoustic and Electromagnetic Scattering Theory, Springer-Verlag, Berlin, Germany, 2nd edition, 1998.

[24] R. A. Kennedy, P. Sadeghi, T. D. Abhayapala, and H. M. Jones, "Intrinsic limits of dimensionality and richness in random multipath fields," IEEE Transactions on Signal Processing, vol. 55, no. 6, pp. 2542-2556, 2007.

[25] E. G. Williams, Fourier Acoustics: Sound Radiation and Nearfield Acoustical Holography, Academic Press, San Deigo, 1999.

[26] M. Abramowitz and I. A. Stegun, Eds., Handbook of Mathematical Functions: with Formulas, Graphs, and Mathematical Tables, Dover Publications, New York, 1965. 